\title{
DIGITAL CONTROL FOR HIGHEST PRECISION ACCELERATOR POWER SUPPLIES
}

\author{
L. Tanner*, F. Jenni*, Paul Scherrer Institute, Villigen, Switzerland
}

\section{Abstract}

Accelerator applications need extremely precise power supplies (PS) for their various magnets. A fully digital control unit enabled identical control hardware for all the devices. In addition, it allowed a high flexibility for the control structures. Adaptations for the different PS are made simple by software. All the drift problems of previous analog solutions were reduced to the precision of one single analog to digital conversion. The achieved current stability and reproducibility is excellent.

\section{INTRODUCTION}

In a Synchrotron Light Source (SLS) electrons are accelerated to very high energy. Over 600 magnet power supplies (PS) with currents up to $1 \mathrm{kA}$ are necessary to control this electron beam. While some of them operate in a single power quadrant only, units with two and four quadrant capability are also needed. All the units are operated hard-switched, with switching-frequencies from $10 \mathrm{kHz}$ up to $100 \mathrm{kHz}$, depending on the PS-ratings. The requirements on the magnetic field, and consequently on the currents, are extremely high: a short term stability down to 10ppm (parts per million) and a long term stability better than $100 \mathrm{ppm}$ is necessary for some of them [1]. In addition, some of the PS require a control bandwidth of more than $1 \mathrm{kHz}$.

Table 1: PS families for the synchrotron light source.

\begin{tabular}{|c|c|c|c|c|c|}
\hline \multirow{2}{*}{$\begin{array}{c}\text { Max. } \\
\text { Curr. }\end{array}$} & \multirow{2}{*}{$\begin{array}{c}\text { Max. } \\
\text { Volt. [V] }\end{array}$} & \multirow{2}{*}{$\begin{array}{c}\text { Power } \\
{[\mathbf{k W}]}\end{array}$} & $\begin{array}{c}\text { Switch } \\
\text { frequ. } \\
{[\mathbf{k H z}]}\end{array}$ & $\begin{array}{c}\text { Short-term } \\
{[\mathbf{p p m}]}\end{array}$ & $\begin{array}{c}\text { Long-term } \\
{[\mathbf{p p m}]}\end{array}$ \\
\hline 950 & $+/-1000$ & 174 & 10 & 10 & 100 \\
\hline 500 & 880 & 430 & 16 & 15 & 100 \\
\hline 120 & 75 & 9 & 100 & 100 & 100 \\
\hline 140 & $+/-120$ & 4.05 & 100 & 100 & 100 \\
\hline 140 & 35 & 4.9 & 100 & 100 & 100 \\
\hline 70 & 145 & 10.2 & 50 & 100 & 100 \\
\hline 120 & 130 & 15.6 & 50 & 100 & 100 \\
\hline 120 & 30 & 3.6 & 100 & 100 & 100 \\
\hline 120 & 60 & 7.2 & 100 & 40 & 100 \\
\hline 120 & 15 & 1.8 & 100 & 500 & 100 \\
\hline$+/-40$ & $+/-40$ & 1.6 & 100 & 100 & 100 \\
\hline$+/-6$ & $+/-110$ & 0.66 & 100 & 500 & 500 \\
\hline$+/-7$ & $+/-20$ & 0.14 & 100 & 15 & 100 \\
\hline
\end{tabular}

The reference values for all PS are generated by the accelerator control system. In previous solutions these values were fed to the analog control circuit of the PS by a precise digital to analog converter (DAC). For supervisory purposes, the measured current was also fed back to the control system via a precise analog to digital converter (ADC).

*lukas.tanner@psi.ch, felix.jenni@psi.ch.
With a digital power supply controller the two conversions, which had to be very precise, can be reduced to a single analog to digital conversion. Drift problems are shifted to the drift of a single voltage reference for the ADC. In addition, the digital control unit allowed a significantly expanded functionality and flexibility. A powerful digital signal processor (DSP controller) and a fast high precision, high stability ADC card were developed in close cooperation with two industrial companies. All the PS of the Swiss Synchrotron Light Source are equipped with this card set, some of them even needing multiple controllers $(+/-1 \mathrm{kV} / 950 \mathrm{~A}$ and $880 \mathrm{~V} /$ 500A PS).

This paper describes in a short form the power electronics' structure of the implemented PS. This is followed by the specifications of the digital control unit and some measurements. The presented system is the first fully digital control of all the PS of an entire accelerator $[2]$.

Figure 1: System overview.

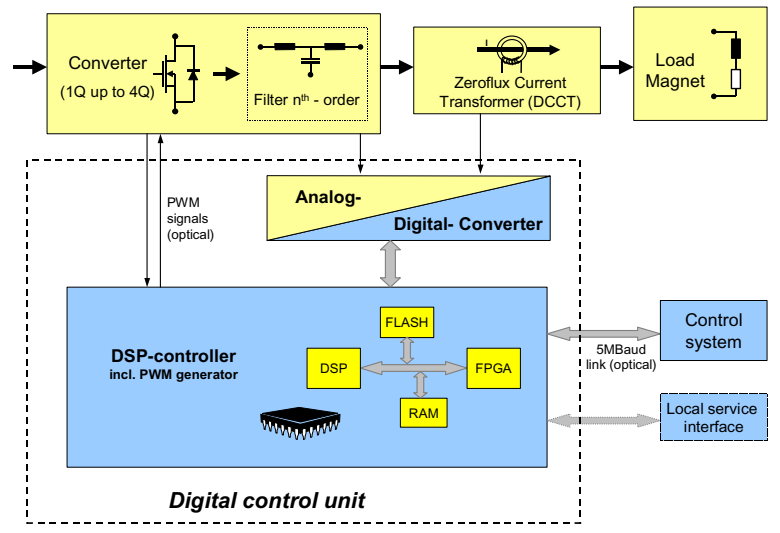

\section{BASIC POWER SUPPLY STRUCTURES}

The accelerator needs over 200 single quadrant PS, some two quadrant PS for positive and negative load voltage and nearly 300 four- quadrant units.

Figure 2: Basic electrical structures.

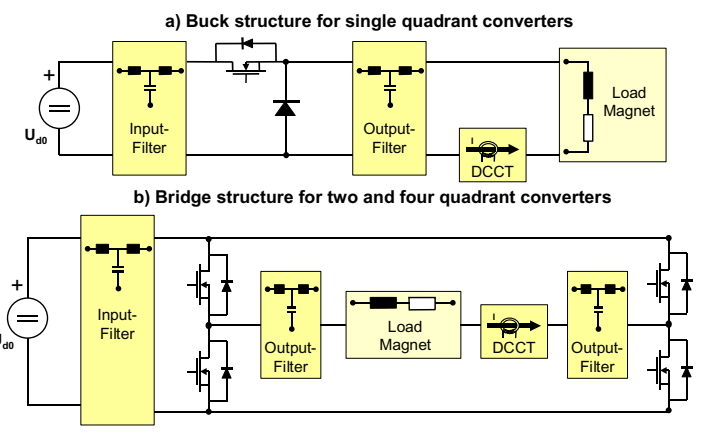




\section{DIGITAL CONTROL UNIT}

The structure of a power supply with the digital control unit, as well as the interface to the control system, corresponds to figure 1 . The control unit enables identical hardware for all the devices and allows a high flexibility for the control structures (For the different PS). Only the control parameters are changing.

\subsection{DSP controller and PWM card}

The core of the DSP- and PWM- card is a powerful $60 \mathrm{MHz}$ clocked digital signal processor with floatingpoint capabilities. The flexibility of this card is extended by a large field-programmable-gate-array (FPGA), which performs all the communications, as well as the pulse width modulation (PWM). 16 digital inputs and 8 outputs are fed via optical couplers. For the control of the power semiconductors and the communication with the control system fibre optic links with 5Mbaud were applied. Multiple cards can be connected together via a fast serial link (30Mbaud), in order to get multiprocessor capability. All software changes and adaptations can be performed by the control system link. For special applications, it is possible to transfer reference current waveforms to the controller cards. These waveforms can then be started by an external trigger signal. For the implemented highresolution PWM two additional new methods had been developed: A rounding correction and a pulse repetition modulation (PRM). With the rounding correction, the limited PWM resolution at high switching-frequencies is compensated (see Figure 4).

Figure 4: Top: Rounding correction influence on PWM resolution. Sinusoidal modulation index between $+/-$ $6.6 \%$. Bottom: Current spectrum up to $1 \mathrm{kHz}$ (Left: rounding on / Right: rounding off).
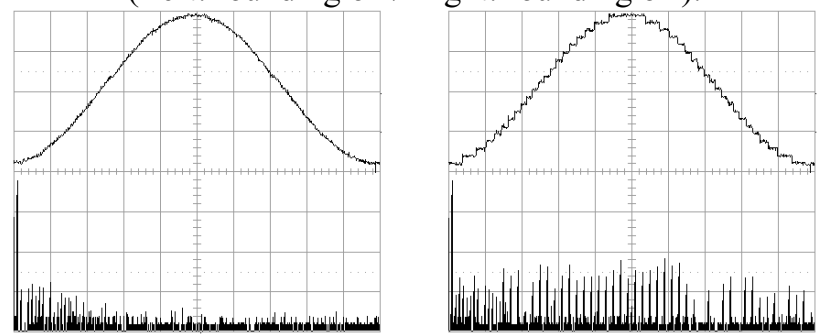

Top: 10ms/DIV, 3.5\%/DIV; Bottom: 0.1kHz/DIV, 20dBm/DIV

The PRM principle enables a current-range down to zero for single quadrant PS. Based on this principle, a digital PWM could be realized, that fulfils the highest demands of accelerator PS [3]. The dynamical qualities of the principle are so high, that the modulation has been demonstrated with a high power audio amplifier.
Figure 3: Simplified functional diagram of the DSP controller and PWM card.

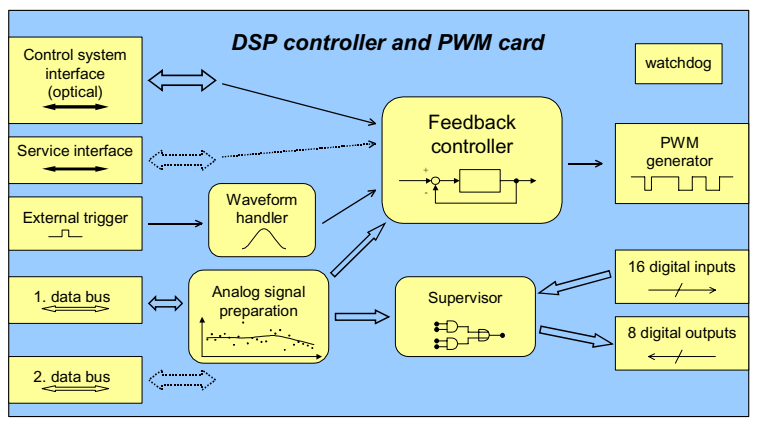

\subsection{AD/DA converter card}

The analog to digital conversion is the crucial point for the precision of the entire controller. Especially for the current measurement (the output property of the PS), a final resolution of less than $10 \mathrm{ppm}$ is required, asking for 17 bits plus sign. This measurement is made by two 16 bit two-channel ADCs with self calibrating capability. At full speed this deliver values at a rate of $200 \mathrm{kHz}$. Additional oversampling technique and the filtering properties of the whole circuit give the desired quality of the current measurement.

Another 4-channel ADC is used for differential measurements of the power supply dc-link and the load voltages. These measurements have reduced requirements compared to the output property; however, they still have a resolution of 16 bits at $50 \mathrm{kHz}$ per channel. For maintenance and trouble shooting two $14 \mathrm{bit} 50 \mathrm{kHz}$ DACchannels are used to monitor internal signals of the controller by an oscilloscope.

Figure 5: Block diagram of AD/DA converter card.

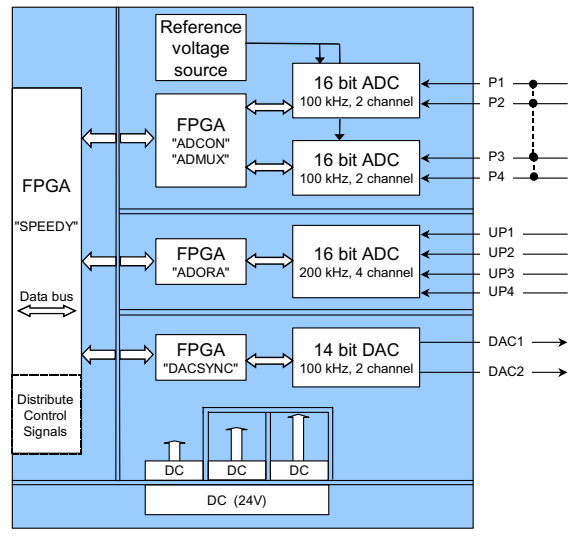

\section{PRECISION OF THE ADC CARD}

For the precision of the PS the ADC card is the crucial element. Therefore, measurements under operating conditions will be presented (Based on a standard card).

\subsection{Resolution and short-term stability $(<60 \mathrm{~s})$}

Figure 6 confirms the high resolution of the converter card. The graphs show the digital value for very small voltage steps $(20 \mu \mathrm{V}=2 \mathrm{ppm})$ at the input. The top graph 
shows the values of 50 samples per second (sps). Each of the samples is the average value of 4000 data points (oversampling). The bottom graph is made of 500sps corresponding to an average of 400 data points. With the developed digital control unit a resolution to $1 \mathrm{ppm}$ is possible.

Figure 6: ADC input signal for 2ppm input voltage steps.
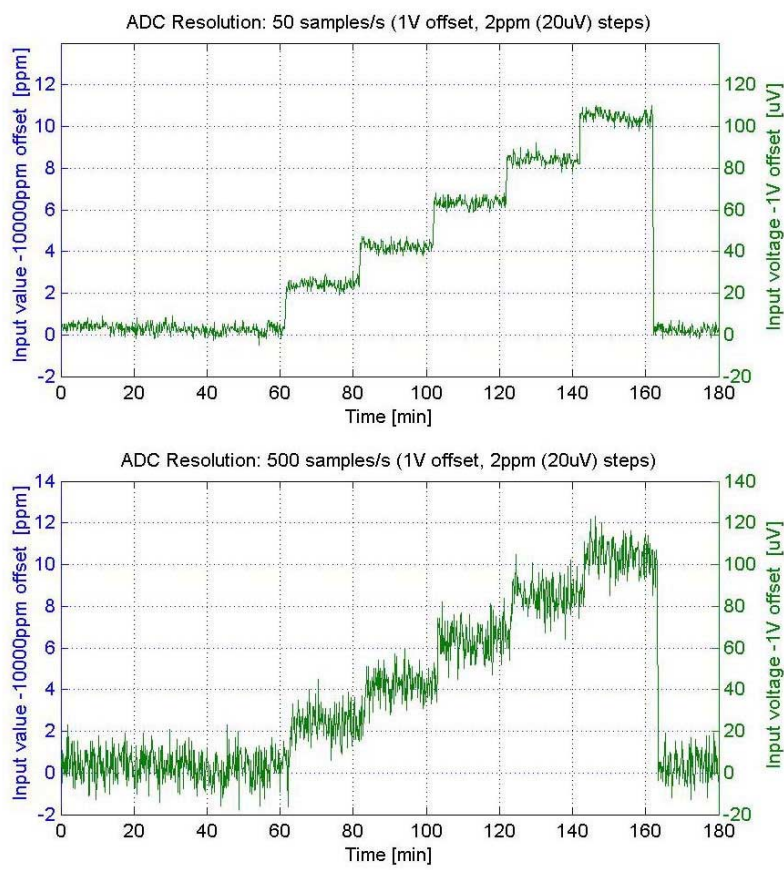

\subsection{Long-term stability}

Figure 7 shows the filtered measured value over 1000 hours. The long-term stability for the first $1000 \mathrm{~h}$ is better than $30 \mathrm{ppm}$.

Figure 7: Long-term measurement over 1000h.

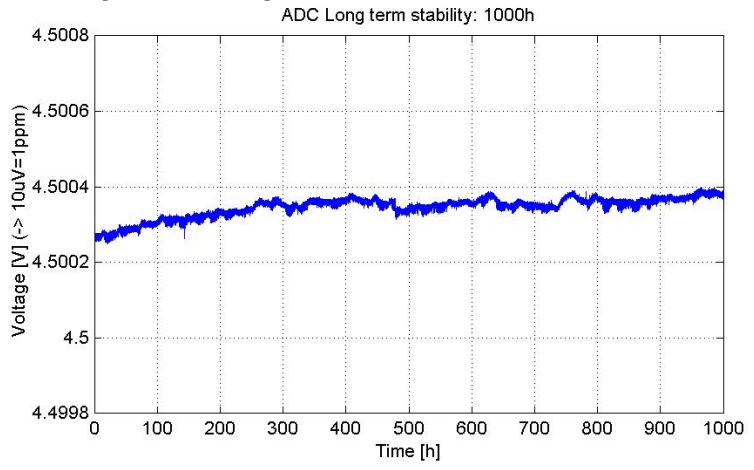

\subsection{Linearity}

The non-linearity of the ADC causes an absolute error, depending on the input voltage. With an error between $+9 \mathrm{ppm}$ and $-14 \mathrm{ppm}$ (see figure 8 ) the analog to digital conversion is very good.
Figure 8: Linearity of analog to digital conversion.

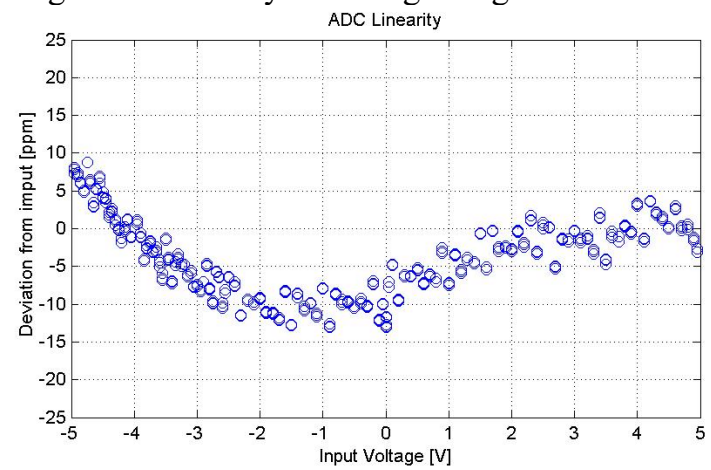

\subsection{Temperature sensitivity}

The precise voltage reference used for ADCs had to be temperature stabilized. Thereby, the temperaturesensitivity in the operation range $\left(20^{\circ} \mathrm{C}\right.$ to $\left.45^{\circ} \mathrm{C}\right)$ could be reduced down to $0.5 \mathrm{ppm} /{ }^{\circ} \mathrm{C}$.

Figure 9: Temperature sensitivity of the $\mathrm{ADC}\left(10^{\circ} \mathrm{C}\right.$ to $\left.50^{\circ} \mathrm{C}\right)$. The dashed line is the ambient temperature.

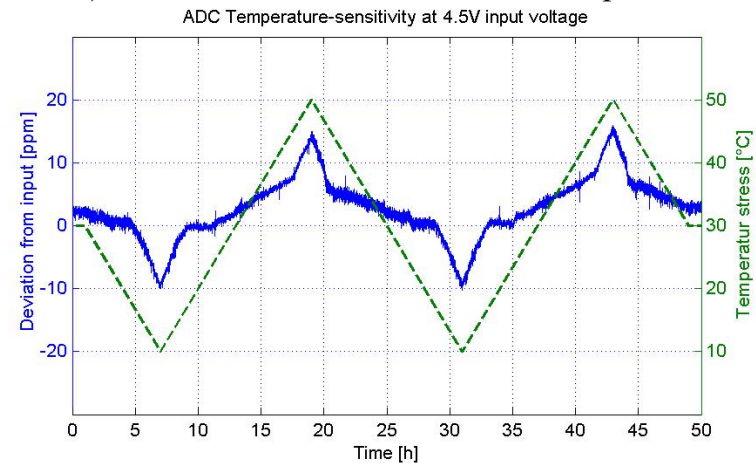

\section{CONCLUSION}

With the realized digital control unit (DSP controller and PWM card, $\mathrm{AD} / \mathrm{DA}$ converter card), it was possible for the first time to handle all the power supplies of an accelerator with a fully digital control. The extreme requirements on precision and dynamic were satisfied. The implementation is based on a sophisticated analog to digital conversion and a powerful control algorithm. At present, nearly 600 units are in operation at the SLS, fulfilling highest requirements.

\section{REFERENCES}

[1] G. Irminger, M. Horvat, F. Jenni, H.U. Boksberger: A $3 \mathrm{~Hz}, 1 \mathrm{MW}$ Bending Magnet Power Supply for the Swiss Light source (SLS); EP2 Forum 98; Electrical Power Technology in European Physics Research.

[2] J. Carwardine, F. Lenkszus: Trends in the Use of Digital Technology for Control and Regulation of Power Supplies; International Conference on Accelerator and Large Experimental Physics Control Systems, 1999.

[3] M. Emmenegger, F. Jenni: A Fully Digital PWM for Highest Precision Power Supplies; European Power Electronics Conference, 2001 